\title{
Molecular detection of Porcine astrovirus in Sichuan Province, China
}

\author{
Yuhan Cai ${ }^{1,2,3 \dagger}$, Wenqi Yin ${ }^{1,2,3 \dagger}$, Yuanchen Zhou ${ }^{1,2,3^{*}}, \mathrm{Bi} \mathrm{Li}^{1,2,3}$, Lun Ai ${ }^{1,2}$, Meng Pan ${ }^{3}$ and Wanzhu Guo ${ }^{4}$
}

\begin{abstract}
Background: Porcine astrovirus (PoAstV) is widely distributed worldwide, and is highly prevalent among piglets with or without diarrhea, existing as at least five distinct lineages (PoAstV1-PoAstV5) within the genus Mamastrovirus. However, our knowledge of the diversity and epidemiology of PoAstV in China is limited.

Results: In this study, fecal samples from 21/120 (17.5 \%) domestic pigs, including 18/100 (18 \%) diarrheic and 3/20 (15\%) healthy pigs, and from 1/9 (11.1\%) healthy wild boars tested in Sichuan Province were positive for PoAstV on reverse transcription-PCR. Of the 22 positive samples, $13.6 \%$ were positive for PoAstV only, whereas $40.9 \%$ also contained Porcine epidemic diarrhea virus (PEDV), $22.7 \%$ also contained porcine group A rotavirus (PRoVA), and $22.7 \%$ also contained PEDV and PRoVA. A phylogenetic analysis of the RdRp gene revealed genetic heterogeneity among the PoAstV sequences and two lineages were detected in this study, with PoAstV-2 predominant. PoAstV-5 was detected in wild boars for the first time.
\end{abstract}

Conclusions: PoAstV infections exist in Sichuan Province regardless of the disease status in the pig population, either alone or in combination with other enteric viruses, and may be associated with diarrhea.

Keywords: Porcine astrovirus, Molecular detection, Phylogenetic analysis

\section{Findings}

Astroviruses (AstVs) are members of the family Astroviridae. They are single-stranded positive-sense RNA viruses with genomes of approximately $7 \mathrm{~kb}$ and spherical, nonenveloped virions of about $30 \mathrm{~nm}$ in diameter. The family Astroviridae is currently separated into two genera, Mamastrovirus (33 species) and Avastrovirus (seven species), which infect mammals and birds, respectively. They are generally associated with either mild or severe enteric disease in mammals, but are also found in healthy animals $[1,2]$. In addition to their intestinal manifestations, recent studies have reported that AstVs can also cause extraintestinal clinical symptoms in humans, minks, pigs, ducks, and goose embryos $[3,4]$.

Porcine astrovirus (PoAstV) was first reported in 1980 in fecal samples from diarrheal pigs, and PoAstV was

\footnotetext{
*Correspondence: abtczyc@163.com

${ }^{\dagger}$ Equal contributors

${ }^{1}$ Livestock and Poultry Biological Products Key Laboratory of Sichuan Province, Huashen Veterinary Biological Products Co., LTD, Chengdu 610299, China

${ }^{2}$ Veterinary Biologicals engineering and technology Research Center of Sichuan Province, Huashen Veterinary Biological Products Co., LTD,

Chengdu 610299, China

Full list of author information is available at the end of the article
}

isolated in culture in 1990. However, the first molecular characterization of PoAstV was not reported until 2001 [5]. Recently, a number of PoAstV isolates have been detected in several countries and characterized, and at least five distinct PoAstV lineages have been identified since PAstV-5 was first identified in fecal samples from slaughtered pigs in Canada in 2009 [6]. To date, PAstV-5 has been identified in domestic pigs, with or without diarrhea, in five countries, including the USA [3, 7], Sweden [4], Canada [6], China [8], and Croatia [2, 9], whereas no study has reported PAstV-5 in wild boars.

However, our knowledge of the diversity and epidemiology of PoAstV in China is limited, and only three studies have reported the genotypes of PAstV distributed across China. One study identified and characterized a PoAstV strain belonging to PoAstV-2 (HQ647383) from diarrheal domestic pigs in 2009 and another detected PoAstV-1 (GQ914773) in healthy domestic pigs in 2008 $[10,11]$. The third study identified and genetically characterized lineages of PAstV-2 (KP747573) and PAstV-5 (KP747574) from healthy domestic piglets in 2015 [8]. In this study, we investigated the infection status and 
genotypes of PoAstV in domestic pigs and wild boars in Sichuan Province, China.

A total of 120 fecal samples from domestic piglets were collected from 76 piggeries across 10 districts of Sichuan Province during the winter of 2014. Of these samples, 100 were taken from diarrheic piglets on 61 farms with epidemic outbreaks of diarrhea and the remaining 20 were taken from asymptomatic piglets from 15 farms with no history of diarrhea. Nine fecal samples from clinically healthy wild boars were collected from wildlife areas located in the northwest of Sichuan Province in December 2014. All the samples were placed into specimen containers and immediately transported to the laboratory at $4{ }^{\circ} \mathrm{C}$ for PCR screening.

The fecal samples were initially examined for the presence of PoAstV with a nested pan-PCR [12] designed to target a conserved region of the RNA-dependent RNA polymerase ( $\mathrm{RdRp}$ ) gene, which has been used previously for the sensitive and specific identification of AstV in mammals, including humans [13], pigs [1, 2, 6, 9, 14], bats [15], birds [16], rodents, shrews, pikas, and weasels [17]. Clinical symptoms of diarrhea are frequently reported to be associated with PEDV, PRoVA, and Transmissible gastroenteritis virus (TGEV) infections in piglets in Sichuan Province, and several papers have described the prevalence of coinfections of PEDV, PRoVA, or TGEV with other enteric pathogens [18, 19]. Therefore, the PoAstV-positive samples were also tested for PEDV, TGEV, and PRoVA with reverse transcription (RT)-PCR, as previously described [18].

The PCR products from PoAstV were cloned into the pMDT-19 simple vector (Takara, Dalian, China) for sequencing (Invitrogen, Carlsbad, CA). All PoAstV sequences were first compared with the BLAST program in NCBI (http://blast.ncbi.nlm.nih.gov/Blast.cgi), and then aligned with other known AstV strains with ClustalW (1.6), using the MEGA 6.06 software. The same software was used to construct a phylogenetic tree from the evolutionary distances between the sequences, using the neighbor-joining (NJ) method and the p-distances of the nucleotide sequences. The clustering stability of the $\mathrm{NJ}$ tree was evaluated with 1000 bootstrap replicates. The sequences of all the PoAstVpositive samples were submitted to GenBank under accession numbers KT440857-KT440878.

Of the 120 domestic pig fecal samples tested, 21 (17.5\%) were positive for PoAstV, a considerably lower overall PoAstV-positive rate than those determined in the Czech Republic (34.2\%) [14], Canada (79.2 \%) [1], and Croatia (89 \%) [2] when the same PCR method was used, but similar to the results when other PCR methods were used, identifying PoAstV prevalence rates of $19.4 \%$ in South Korea [20] and $20.8 \%$ in Germany [21]. The positive rates in non-diarrheic and diarrheic pig fecal samples were $15 \%(3 / 20)$ and $18 \%(18 / 100)$, respectively. Only one wild boar tested positive for PoAstV $(11.1 \%, 1 / 9)$. These results suggest that PoAstV is present in Sichuan Province, regardless of the disease status of domestic pigs. The low prevalence of PoAstV in wild boars might be partly attributable to the fact that wild pigs are generally more resistant to many diseases than are domesticated pigs and partly to the limited number of samples tested.

Of the 22 PoAstV-positive samples, three (13.6\%) were positive for PoAstV alone, and nine (40.9 \%) were positive for PoAstV and PEDV, five (22.7 \%) for PoAstV and PRoVA, and five $(22.7 \%)$ for PoAstV, PEDV, and PRoVA (Additional file 1: Table S1). No co-infection with PoAstV and TGEV was detected in this study. Based on these co-infection rates, we infer that PoAstVinfected piglets are probably always co-infected with PEDV and/or PRoVA, consistent with previous studies [18, 20-23]. As well as associations between PoAstV and PRoVA or PEDV, co-infections of PoAstV and other diarrheal pathogens, such as TGEV, porcine bocavirus, porcine torovirus, porcine enterovirus 9, porcine sapovirus, or porcine norovirus, have been observed in previous studies $[18,21-24]$. However, the exact role of the AstVs, whether alone or in combination with other enteric viruses, in causing diarrhea in pigs is not yet clear.

Genetic typing has been very useful in tracing the evolution and spread of viruses. In this study, we conducted a phylogenetic analysis of a region of the RdRp gene in prototypical PoAstVs, human AstVs, and other AstVs. On the phylogenetic tree (Fig. 1), the PoAstVs were divided into five distinct lineages (PoAstVs 1-5), and some of the PoAstVs are phylogenetically related to other animal and human AstV strains. Recent studies have shown evidence of possible recombination events between porcine and human AstVs [25] and interspecies recombination between porcine and deer AstVs [6]. These findings suggest the zoonotic potential of PoAstV strains.

The 22 PoAstV sequences formed two lineages in the pig population of Sichuan Province (Fig. 1, Additional file 2: Table S2). Twelve strains, comprising two isolates from healthy domestic pigs and 10 isolates from domestic pigs with diarrhea, potentially belonged to PoAstV-2 (54.5\%), sharing 82-99.7\% nucleotide identity, and they formed a single lineage on the phylogenetic tree. Ten strains (45.5\%), including one isolate from a healthy domestic pig, eight isolates from diarrheic domestic pigs, and one isolate from a healthy wild boar, clustered with PoAstV-5, sharing quite high nucleotide similarities of 95-99.4 \%. On the phylogenetic tree (Fig. 1), PoAstV-5 has two branches. The Chinese PoAstV-5 strains localized on the same branch as strain JX519277 detected in Croatia in 2011, with 90.5-93.5 \% nucleotide identity, and JX519277 was about $73 \%$ identical to the strains on 


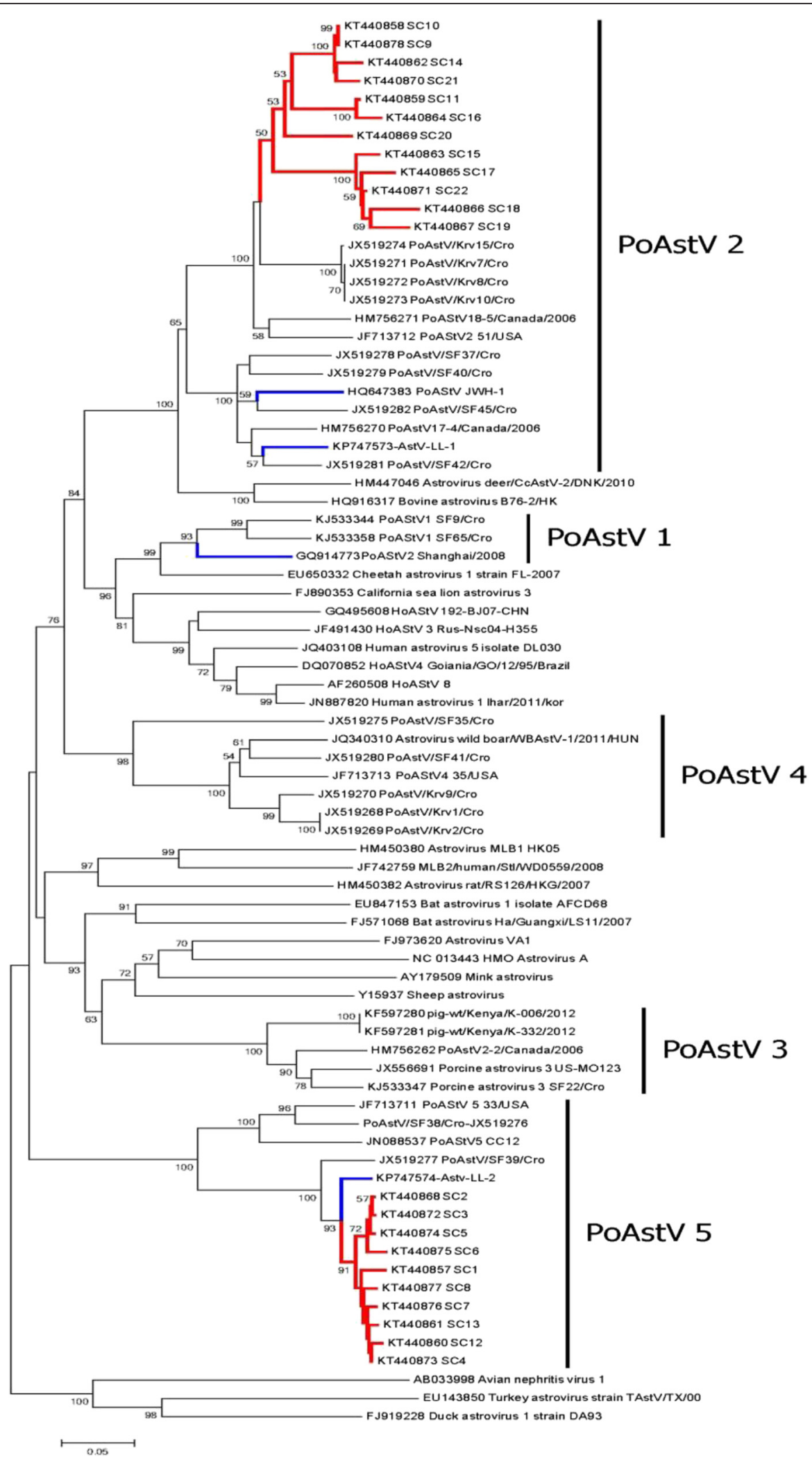

Fig. 1 (See legend on next page.) 
(See figure on previous page.)

Fig. 1 Phylogenetic relationships between the RdRp genes (422 bp) of the porcine astrovirus sequences identified in this study (shown in bold and red) and in previous studies in China (bold and blue) and selected reference sequences (GenBank accession numbers shown with the taxa). The tree was constructed with the neighbor-joining method using the p-distance substitution model, with 1000 bootstrap replicates and a cut-off value of $70 \%$, with the MEGA 6.06 software. The scale bar represents the number of nucleotide substitutions per site

the other branch of PoAstV-5 (JF713711, JX519276, and JN088537). These results suggest that the Chinese PoAstV-5 strains are closely related to JX519277, providing new data for the analysis of PoAstV-5 evolution. However, the origin of the PoAstV-5 strains circulating in China requires further investigation.

Previous studies have reported four PoAstV strains in China: one PoAstV-1 sequence (GQ914773), two PoAstV-2 sequences (HQ647383 and KP747573), and one PoAstV-5 sequence (KP747574). Like previous studies, we also identified PAstV-2 and PAstV-5 in the pig population in Sichuan Province (Fig. 1). The PoAstV-2 sequences detected in our study displayed similarities of 74 and $73.6 \%$ with diversities of 3.4 and $3.5 \%$ to HQ647383 and KP747573, respectively. However, the PoAstV-5 sequences identified in our study shared nucleotide homologies of 94.3-96.7 \% with KP747574. These results indicate that three genotypes, PAstV-1, PAstV-2, and PAstV-5, occur in China, with possible antigenic variations among the PoAstVs, as have been reported in several other studies [1,24, 25]. This is the first time that PoAstV- 5 has been detected in wild boars anywhere in the world.

To date, PAstV1-PAstV5 have all been detected in fecal samples from diarrheal or apparently healthy pigs $[1,3,6,8]$. PAstV5 can also cause congenital tremors [4], and PAstV2 and PAstV4 are found in the blood of pigs [9]. Therefore, more work is required to determine why AstVs are found in both healthy and diarrheic pigs and the clinical manifestations of PAstV infections must be clarified.

In conclusion, this study has demonstrated the presence of PoAstV in domestic pigs and wild boars in Sichuan Province, China, and co-infections of PoAstV with PEDV and/or PRoVA. A phylogenetic analysis of the RdRp gene sequence revealed that PoAstV-2 and PoAstV-5 were isolated in this study. To our knowledge, PoAstV-5 was detected in wild boars for the first time anywhere in the world. PoAstVs play an important role in the evolution and ecology of the Astroviridae, and recombination analyses are required to clarify the evolution of PoAstV in Sichuan Province.

\section{Additional files}

Additional file 1: Table S1. Occurrence of PoAstV with other enteric viruses. (DOC $30 \mathrm{~kb}$ )

Additional file 2: Table S2. Characterization of PoAstV identified in the present study. (DOC $37 \mathrm{~kb}$ )
Competing interests

The authors declare no conflict of interest.

\section{Authors' contributions}

Yuhan Cai, Wenqi Yin, Wanzhu Guo and Yuancheng Zhou conceived and designed the experiments. Yuhan Cai, Bi Li, and Yuanchen Zhou performed the experiments. Yuhan Cai, Meng Pan, Lun Ai, and Yuanchen Zhou analyzed the data. Yuhan Cai and Yuancheng Zhou wrote the manuscript. All authors read and approved the final manuscript.

\section{Acknowledgements}

This study was supported by Transformation of Scientific and Technological Achievements Program of Sichuan Province (2014CC056), and Science and Technology Support Program of Sichuan province (2013NZ0016 and 2015NZ0105)

\section{Author details}

'Livestock and Poultry Biological Products Key Laboratory of Sichuan Province, Huashen Veterinary Biological Products Co., LTD, Chengdu 610299, China.

${ }^{2}$ Veterinary Biologicals engineering and technology Research Center of Sichuan Province, Huashen Veterinary Biological Products Co., LTD,

Chengdu 610299, China. Institute of Animal Nutrition, Sichuan Academy of Animal Science, Chengdu 610299, China. ${ }^{4}$ Animal Biotechnology Center, College of Veterinary Medicine, Sichuan Agricultural University, Chengdu 611134, China.

Received: 17 October 2015 Accepted: 30 December 2015

Published online: 06 January 2016

\section{References}

1. Luo Z, Roi S, Dastor M, Gallice E, Laurin M-A, L'Homme Y. Multiple novel and prevalent astroviruses in pigs. Vet Microbiol. 2011;149(3):316-23.

2. Brnić $D$, Jemeršić $L$, Keros T, Prpić J. High prevalence and genetic heterogeneity of porcine astroviruses in domestic pigs. Vet J. 2014; 202(2):390-2.

3. Xiao C-T, Giménez-Lirola LG, Gerber PF, Jiang Y-H, Halbur PG, Opriessnig T. Identification and characterization of novel porcine astroviruses (PAstVs) with high prevalence and frequent co-infection of individual pigs with multiple PAstV types. J Gen Virol. 2013;94(Pt 3):570-82.

4. Blomström A-L, Ley C, Jacobson M. Astrovirus as a possible cause of congenital tremor type All in piglets? Acta Vet Scand. 2014;56(1):82.

5. Jonassen $C M$, Jonassen $T \varnothing$, Saif $Y M$, Snodgrass DR, Ushijima H, Shimizu M, et al. Comparison of capsid sequences from human and animal astroviruses. J Gen Virol. 2001;82(5):1061-7.

6. Laurin M-A, Dastor M, L'Homme Y. Detection and genetic characterization of a novel pig astrovirus: relationship to other astroviruses. Arch Virol. 2011; 156(11):2095-9.

7. Shan T, Li L, Simmonds P, Wang C, Moeser A, Delwart E. The fecal virome of pigs on a high-density farm. J Virol. 2011;85(22):11697-708.

8. Li J-s, Li M-Z, Zheng L-s, Liu N, Duan Z-j. Identification and genetic characterization of two porcine astroviruses from domestic piglets in China. Archives of virology. 2015;160(12):3079-3084

9. Brnić D, Prpić J, Keros T, Roić B, Starešina V, Jemeršić L. Porcine astrovirus viremia and high genetic variability in pigs on large holdings in Croatia. Infect Genet Evol. 2013;14:258-64.

10. Lan D, Ji W, Shan T, Cui L, Yang Z, Yuan C, et al. Molecular characterization of a porcine astrovirus strain in China. Arch Virol. 2011;156(10):1869-75.

11. Shan T, Wang C, Tong W, Zheng H, Hua X, Yang S, et al. Complete genome of a novel porcine astrovirus. J Virol. 2012;86(24):13820-1.

12. Chu D, Poon L, Guan Y, Peiris J. Novel astroviruses in insectivorous bats. J Virol. 2008;82(18):9107-14. 
13. Kapoor A, Li L, Victoria J, Oderinde B, Mason C, Pandey P, et al. Multiple novel astrovirus species in human stool. J Gen Virol. 2009; 90(12):2965-72.

14. Dufkova L, Scigalkova I, Moutelikova R, Malenovska H, Prodelalova J. Genetic diversity of porcine sapoviruses, kobuviruses, and astroviruses in asymptomatic pigs: an emerging new sapovirus GIII genotype. Arch Virol. 2013;158(3):549-58.

15. Xiao J, Li J, Hu G, Chen Z, Wu Y, Chen Y, et al. Isolation and phylogenetic characterization of bat astroviruses in southern China. Arch Virol. 2011; 156(8):1415-23.

16. Chu DK, Leung $C Y$, Perera HK, Ng EM, Gilbert M, Joyner PH, et al. A novel group of avian astroviruses in wild aquatic birds. J Virol. 2012 86(24):13772-8

17. Hu B, Chmura AA, Li J, Zhu G, Desmond JS, Zhang Y, et al. Detection of diverse novel astroviruses from small mammals in China. J Gen Virol. 2014; 95(Pt 11):2442-9.

18. Zhou Y, Chen L, Zhu L, Xu Z. Molecular Detection of Porcine Torovirus in Piglets with Diarrhea in Southwest China. Scientific World Journal. 2013; 2013:984282.

19. Zhou L, Wei H, Zhou Y, Xu Z, Zhu L, Horne J. Molecular epidemiology of Porcine torovirus (PToV) in Sichuan Province, China: 2011-2013. Virol J. 2014;11(1):1-9.

20. Lee M-H, Jeoung H-Y, Park H-R, Lim J-A, Song J-Y, An D-J. Phylogenetic analysis of porcine astrovirus in domestic pigs and wild boars in South Korea. Virus Genes. 2013:46(1):175-81.

21. Machnowska P, Ellerbroek L, Johne R. Detection and characterization of potentially zoonotic viruses in faeces of pigs at slaughter in Germany. Vet Microbiol. 2014;168(1):60-8.

22. Monini M, Di Bartolo I, laniro G, Angeloni G, Magistrali CF, Ostanello F, et al. Detection and molecular characterization of zoonotic viruses in swine fecal samples in Italian pig herds. Arch Virol. 2015;160(10):2547-56.

23. Zhang B, Tang C, Yue H, Ren Y, Song Z. Viral metagenomics analysis demonstrates the diversity of viral flora in piglet diarrhoeic faeces in China. J Gen Virol. 2014;95(Pt 7):1603-11.

24. Mor SK, Chander Y, Marthaler D, Patnayak DP, Goyal SM. Detection and molecular characterization of Porcine astrovirus strains associated with swine diarrhea. J Vet Diagn Invest. 2012;24(6):1064-7.

25. Ulloa JC, Gutiérrez MF. Genomic analysis of two ORF2 segments of new porcine astrovirus isolates and their close relationship with human astroviruses. Can J Microbiol. 2010;56(7):569-77.

\section{Submit your next manuscript to BioMed Central and we will help you at every step:}

- We accept pre-submission inquiries

- Our selector tool helps you to find the most relevant journal

- We provide round the clock customer support

- Convenient online submission

- Thorough peer review

- Inclusion in PubMed and all major indexing services

- Maximum visibility for your research

Submit your manuscript at www.biomedcentral.com/submit

C Biomed Central 